\title{
Paradigm shift in local consolidative therapy for oligometastatic non-small cell lung cancer: a meta-analysis
}

\author{
Christopher Cao ${ }^{1,2,3,4}$, Adam Chakos ${ }^{3}$, Allen Guo ${ }^{3}$, John J. Park ${ }^{5}$, Tajalli Saghaie ${ }^{6}$, Prunella Blinman \\ ${ }^{1}$ Department of Cardiothoracic Surgery, Royal Prince Alfred Hospital, Sydney University, Sydney, Australia; ${ }^{2}$ Department of Cardiothoracic Surgery, \\ Concord Repatriation Hospital, Sydney, Australia; ${ }^{3}$ Department of Cardiothoracic Surgery, Collaborative Research Group, Sydney, Australia; \\ ${ }^{4}$ Department of Cardiothoracic Surgery, Lifehouse Cancer Hospital, Sydney, Australia; ${ }^{5}$ Faculty of Medicine and Health Sciences, Macquarie University, \\ Sydney, Australia; ${ }^{6}$ Department of Respiratory Medicine, ${ }^{7}$ Department of Medical Oncology, Concord Repatriation Hospital, Sydney, Australia \\ Correspondence to: Christopher Cao. Professor, Department of Cardiothoracic Surgery, Royal Prince Alfred Hospital, Sydney University, 50 \\ Missenden Road, Camperdown, Sydney, NSW 2050, Australia. Email: drchriscao@gmail.com. \\ Provenance: This is an invited article commissioned by the Section Editor Dr. Song Xu (Department of lung cancer surgery, Tianjin Medical \\ University General Hospital; Tianjin Key Laboratory of Lung Cancer Metastasis and Tumor Microenvironment, Lung Cancer Institute, Tianjin, \\ China). \\ Comment on: Gomez DR, Tang C, Zhang J, et al. Local Consolidative therapy vs. maintenance therapy or observation for patients with oligometastatic \\ non-small-cell lung cancer: long-term results of a multi-institutional, phase II, randomized study. J Clin Oncol 2019;37:1558-65.
}

Submitted Sep 14, 2019. Accepted for publication Sep 26, 2019.

doi: 10.21037/atm.2019.09.147

View this article at: http://dx.doi.org/10.21037/atm.2019.09.147

Should patients with oligometastatic non-small cell lung cancer (NSCLC) undergo local consolidative therapy (LCT)? That was the question posed by Gomez and colleagues, who should be congratulated on their randomized controlled trial originally published in 2016 in Lancet Oncology (1). The trial selected patients with histologically-proven stage IV NSCLC with $\leq 3$ sites of disease after first-line systemic therapy, prior to randomization to either LCT and maintenance therapy (MT) or maintenance therapy/observation (MTO) alone. Randomization was balanced dynamically according to the number of metastases, response to initial therapy, central nervous system metastases, nodal status, and EGFR/ALK status. The primary endpoint of the trial was progression-free survival (PFS). Although the initial aim was to randomize 94 patients, the study was terminated prematurely after treatment of 49 patients due to detection of a significant superiority in PFS for the LCT group (11.9 vs. 3.9 months; $\mathrm{P}=0.005$ ). Local consolidative treatment regimens to the primary and metastatic lesions included hypofractionated radiotherapy, surgery/radiation, chemoradiation, or surgery alone, to treat all sites of disease. MT provided in both arms included pemetrexed, targeted therapy agents, and bevacizumab. The authors concluded in the 2016 publication that aggressive local therapy should be considered in selected patients with oligometastatic stage IV NSCLC, particularly as the benefit of increased PFS was achieved without increasing the incidence of grade $\geq 3$ adverse events (1). However, it should be noted that the median follow-up at the time was only 12.4 months from the time of randomization due to premature termination by the Data Safety Monitoring Committee.

With prolonged follow-up beyond 38 months, Gomez and colleagues recently presented their updated data in the Fournal of Clinical Oncology (2). The previously identified key finding persisted, with a significantly longer median PFS in the LCT group compared to the MTO group (14.2 vs. 4.4 months, $\mathrm{P}=0.022$ ). Furthermore, median overall survival was also significantly longer in the LCT group (41.2 vs. 17.0 months, $\mathrm{P}=0.017)$. Of additional interest, 15 out of 39 patients who experienced disease progression and then underwent LCT achieved a longer overall survival compared to patients who had disease progression and did not have further LCT. In other words, early LCT was better than no LCT, but late or 'salvage' LCT after disease progression was still better than no LCT at all. These findings were supported by another recent trial by Iyengar and colleagues, who randomized patients to stereotactic body radiation therapy (SBRT) and MT versus MT alone (3). In contrast to the Gomez study, oligometastatic disease was 


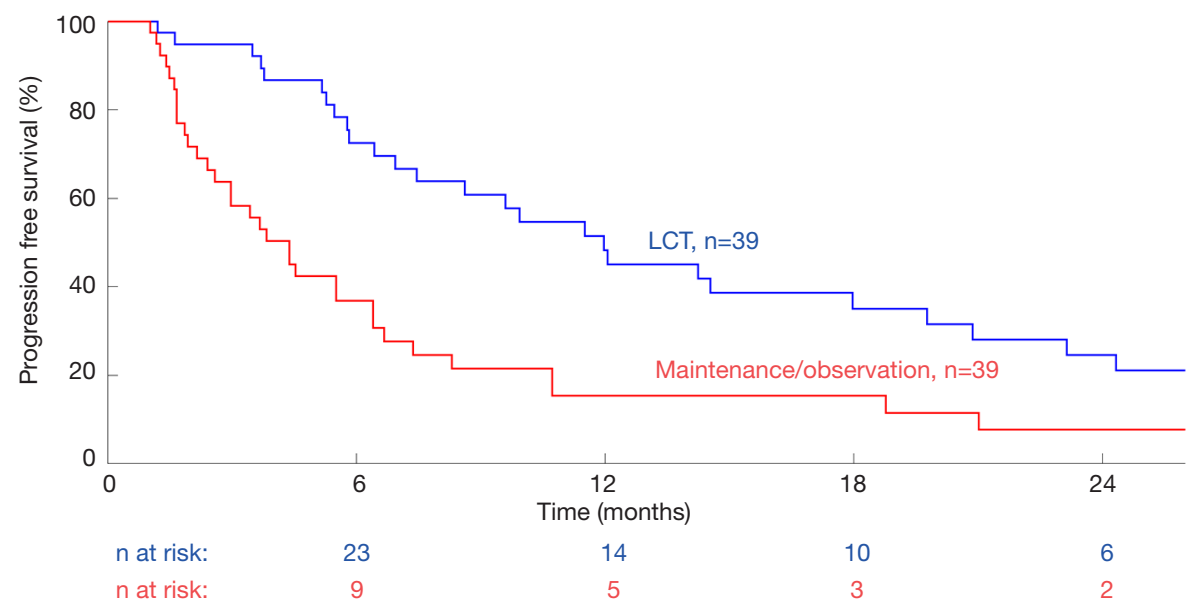

Figure 1 PFS for LCT and MT versus MT alone for patients with oligometastatic NSCLC. Curves generated by aggregation of published data from randomized controlled trials. PFS, progression-free survival; LCT, local consolidative therapy; MT, maintenance therapy; NSCLC, non-small cell lung cancer.

Table 1 Summary of study characteristics for randomized controlled trials comparing LCT and MT versus MT alone in patients with oligometastatic stage IV NSCLC

\begin{tabular}{lllllll}
\hline Author, year & Institution & $\begin{array}{l}\text { Enrolment } \\
\text { period }\end{array}$ & $\begin{array}{l}\text { Follow-up } \\
\text { period }\end{array}$ & $\begin{array}{l}\text { LCT } \\
(\mathrm{n})\end{array}$ & $\begin{array}{l}\text { MT } \\
(\mathrm{n})\end{array}$ & LCT regimen \\
\hline Gomez, 2019 & $\begin{array}{l}\text { MD Anderson Cancer Center, USA; } \\
\text { London Health Sciences Center, Canada; } \\
\text { Colorado School of Medicine, USA }\end{array}$ & $2012-2016$ & 38.8 months & 25 & 24 & Surgery or radiation \\
lyengar, 2017 & $\begin{array}{l}\text { University of Texas Southwestern Medical } \\
\text { Center, USA }\end{array}$ & $2014-2016$ & 9.6 months & 14 & 15 & $\begin{array}{l}\text { SBRT followed by } \\
\text { maintenance chemotherapy }\end{array}$ \\
\hline
\end{tabular}

LCT, local consolidative therapy; MT, maintenance therapy; NSCLC, non-small cell lung cancer; SBRT, stereotactic body radiation therapy.

defined as $\leq 5$ lesions, and patients with targetable mutations were excluded from enrolment. Once again, the trial was terminated early after interim analysis demonstrated significant benefit in PFS in the LCT treatment arm (9.7 vs. 3.5 months, $\mathrm{P}=0.01$ ), without any increase in toxicities. Although this prospective randomized trial was limited by its sample size to analyze overall survival, larger retrospective series have reported improved median overall survival in patients who underwent LCT, particularly those with limited intrathoracic burden, lack of bony disease, and non-squamous histopathology (4).

In order to summarize the current Level 1 evidence, we performed a meta-analysis using all the identified randomized data in the existing literature. KaplanMeier curves from two randomized controlled trials were aggregated using techniques developed by Guyot and colleagues for conducting secondary analysis of survival data $(2,3,5)$. This method involves individual patient data being back-calculated from digitized survival curves while considering time-matched numbers-at-risk. Individual patient data was then combined in a Kaplan-Meier model to generate combined overall survival curves. Hazard ratios (HR) between survival curves were calculated using a Cox proportional hazard model. The threshold for significance was taken as $\mathrm{P}<0.05$. These calculations were performed using R ( R foundation for statistical computing, Vienna, Austria), and Matlab R2017b (The MathWorks, Natick, Massachusetts, USA), and are presented in Figure 1. This demonstrated a significantly increased PFS curve for LCT and MT versus MT alone [HR, 0.42; 95\% confidence interval (CI), 0.24-0.69; $\mathrm{P}=0.001]$. A summary of study characteristics is presented in Table 1, and it should be noted that a third randomized trial was excluded from analysis due to a lack of individualized data for patients with primary 
lung cancer (6).

How will these findings affect clinical practice in the contemporary era? Since the closure of enrolment in the Gomez trial in January 2016, the introduction of immunotherapy has led to a paradigm shift in the treatment of advanced stage NSCLC $(7,8)$. With prolonged PFS and lower toxicity profile, it is foreseeable that a higher proportion of patients with oligometastatic NSCLC treated by immunotherapeutic agents will achieve stable disease and become eligible candidates for LCT. Similarly, targeted therapy has continued to evolve with maturing data and emergence of new drugs. Gomez and colleagues have commenced enrolment in a phase II randomized trial comparing osimertinib with or without LCT (ClinicalTrials.gov identifier: NCT03410043). Regarding local consolidative treatment modalities, the popularization of SBRT and minimally invasive thoracic surgery have improved the tolerability and efficacy of LCT in the contemporary era $(9,10)$. Compared to traditional radiotherapy, SBRT delivers fewer fractions of high-dose radiation with increased precision and higher biologically effective dose, resulting in reduced toxicity and improved local control (11). Conventional and robotic videoassisted thoracoscopic surgery have both been associated with reduced perioperative morbidity whilst maintaining long-term oncological efficacy when compared to the traditional thoracotomy approach in the treatment of NSCLC $(10,12,13)$. With these advancements in systemic therapy and LCT, it is hoped that a greater proportion of patients diagnosed with stage IV NSCLC will be eligible for lower risk and more effective local therapies to improve their PFS and overall survival. Future studies should be consistent in the definition of oligometastatic disease, aim to identify important prognostic factors that could help to refine patient selection, and further assess quality of life and cost effectiveness outcomes. Discussion and consideration of LCT for this growing cohort of patients in a multidisciplinary team setting will be critical to ensure the optimal treatment for individual patients.

\section{Acknowledgments}

None.

\section{Footnote}

Conflicts of Interest: The authors have no conflicts of interest to declare.
Ethical Statement: The authors are accountable for all aspects of the work in ensuring that questions related to the accuracy or integrity of any part of the work are appropriately investigated and resolved.

\section{References}

1. Gomez DR, Blumenschein GR Jr, Lee JJ, et al. Local consolidative therapy versus maintenance therapy or observation for patients with oligometastatic non-smallcell lung cancer without progression after first-line systemic therapy: a multicentre, randomised, controlled, phase 2 study. Lancet Oncol 2016;17:1672-82.

2. Gomez DR, Tang C, Zhang J, et al. Local Consolidative therapy vs. maintenance therapy or observation for patients with oligometastatic non-small-cell lung cancer: long-term results of a multi-institutional, phase II, randomized study. J Clin Oncol 2019;37:1558-65.

3. Iyengar P, Wardak Z, Gerber DE, et al. Consolidative radiotherapy for limited metastatic non-small-cell lung cancer: a phase 2 randomized clinical trial. JAMA Oncol 2018;4:e173501.

4. Mitchell KG, Farooqi A, Ludmir EB, et al. Improved overall survival with comprehensive local consolidative therapy in synchronous oligometastatic non-small-cell lung cancer. Clin Lung Cancer 2019. [Epub ahead of print].

5. Guyot P, Ades AE, Ouwens MJ, et al. Enhanced secondary analysis of survival data: reconstructing the data from published Kaplan-Meier survival curves. BMC Med Res Methodol 2012;12:9.

6. Palma DA, Olson R, Harrow S, et al. Stereotactic ablative radiotherapy versus standard of care palliative treatment in patients with oligometastatic cancers (SABRCOMET): a randomised, phase 2, open-label trial. Lancet 2019;393:2051-8.

7. Gandhi L, Rodríguez-Abreu D, Gadgeel S, et al. Pembrolizumab plus chemotherapy in metastatic nonsmall-cell lung cancer. N Engl J Med 2018;378:2078-92.

8. Reck M, Rodríguez-Abreu D, Robinson AG, et al. Pembrolizumab versus chemotherapy for pd-11positive non-small-cell lung cancer. N Engl J Med 2016;375:1823-33.

9. Cao C, Wang D, Chung C, et al. A systematic review and meta-analysis of stereotactic body radiation therapy versus surgery for patients with non-small cell lung cancer. J Thorac Cardiovasc Surg 2019;157:362-73.e8.

10. Cao C, Manganas C, Ang SC, et al. A systematic review 
and meta-analysis on pulmonary resections by robotic video-assisted thoracic surgery. Ann Cardiothorac Surg 2012;1:3-10.

11. Palma D, Visser O, Lagerwaard FJ, et al. Impact of introducing stereotactic lung radiotherapy for elderly patients with stage I non-small-cell lung cancer: a population-based time-trend analysis. J Clin Oncol 2010;28:5153-9.

Cite this article as: Cao C, Chakos A, Guo A, Park JJ, Saghaie T, Blinman P. Paradigm shift in local consolidative therapy for oligometastatic non-small cell lung cancer: a metaanalysis. Ann Transl Med 2019;7(Suppl 8):S320. doi: 10.21037/ atm.2019.09.147
12. Cao C, Manganas C, Ang SC, et al. A meta-analysis of unmatched and matched patients comparing videoassisted thoracoscopic lobectomy and conventional open lobectomy. Ann Cardiothorac Surg 2012;1:16-23.

13. Cerfolio RJ, Ghanim AF, Dylewski M, et al. The longterm survival of robotic lobectomy for non-small cell lung cancer: a multi-institutional study. J Thorac Cardiovasc Surg 2018;155:778-86. 